\title{
Metabolite Imaging Using Matrix-Enhanced Surface-Assisted Laser Desorption/Ionization Mass Spectrometry (ME-SALDI-MS)
}

\author{
Qiang Liu, Yongsheng Xiao, Coral Pagan-Miranda, ${ }^{*}$ Yu Matthew Chiu, ${ }^{+}$ \\ and Lin He \\ Department of Chemistry, North Carolina State University, Raleigh, North Carolina, USA
}

We describe here the use of a hybrid ionization approach, matrix-enhanced surface-assisted laser desorption/ionization mass spectrometry (ME-SALDI-MS) in bioimaging. ME-SALDI combines the strengths of traditional matrix-assisted laser desorption/ionization (MALDI) and SALDI and enables successful MS imaging of low-mass species with improved detection sensitivity. Using 1,2-dimyristoyl-sn-glycero-3-phosphocholine (DMPC) as the MS standard, MS performances of MALDI, SALDI, and ME-SALDI are systematically compared. The analyte desorption and ionization mechanism in ME-SALDI is qualitatively speculated based on the observation of significantly reduced matrix background and improved survival yields of molecular ions. Improvements in detection sensitivity of low-mass species using ME-SALDI over MALDI in imaging are demonstrated with mouse heart and brain tissues. (J Am Soc Mass Spectrom 2009, 20, 80-88) (c) 2009 Published by Elsevier Inc. on behalf of American Society for Mass Spectrometry

$\mathrm{B}$ y providing both the chemical identities and the spatial distribution of hundreds of components in biological systems simultaneously without pretagging distinctive labels, imaging mass spectrometry (IMS) has created new research opportunities in many fields [1-7]. However, despite rapid developments in the field of IMS, several fundamental issues remain to be addressed. In particular, analyte ionization efficiency is in urgent need of improvement, giving its importance in enhancing detection sensitivity of less-abundant bioactive species as the imaging footprint decreases [8].

Technology development rapidly advances when two complementary methodologies are combined to overcome the inherent difficulties encountered by each one. It is especially true in mass spectrometry (MS), where hybrid approaches constantly change the landscape of the field. Indeed, in addition to numerous hyphened mass analyzers, hybrid mass ionization techniques - such as matrix-enhanced secondary ion mass spectrometry (ME-SIMS) [3, 9], desorption electrospray ionization (DESI) [10], electrospray-assisted laser desorption ionization (ELDI) [11], laser desorption atmospheric-pressure chemical ionization (LD-APCI) [12], and matrix-assisted laser desorption electrospray ionization (MALD-ESI) [13] — have together augmented the overall applicability of MS in solving increasingly

Address reprint requests to: Prof. Lin He, North Carolina State University, Department of Chemistry, Raleigh, NC 27695. E-mail: lin_he@ncsu.edu

* Present address: University of Puerto Rico, Department of Chemistry, Rio Piedras Campus, San Juan, PR 00931.

† Present address: Lab Corporation of America, Durham, NC 27703. complex biological problems. The concept of mixing photon-absorbing solid materials (metal nanoparticles and graphite particles, etc.) with nonabsorbing liquid (such as glycerol derivative) has also been demonstrated to improve upper mass limit, where glycerol mainly serves to improve the mixing of analytes with suspended particles and to dissipate excess heating energy [14-17]. Mixing the conventional solid matrixassisted laser desorption/ionization (MALDI) matrix with a porous Si surface has been reported where the surface serves as a solid-phase extraction medium to concentrate selected analytes [18].

Adapting a similar concept, we describe here the use of a hybrid ionization method, i.e., matrix-enhanced SALDI (ME-SALDI), in two dimensional (2-D) IMS with the aim of improving ionization efficiency of low-mass species. ME-SALDI combines valuable attributes from both conventional MALDI and SALDI methods. In particular: (1) the introduction of the solid conventional MALDI matrix, such as CHCA, to SALDI takes advantage of its strong proton-donating capability and protects analytes from deconstructive overheating [19, 20]; (2) the presence of porous surface absorbs incident photons more effectively than conventional MALDI matrices. The application of ME-SALDI in MS imaging is demonstrated for the first time in this report. It provides a complementary tool to widely used MALDI IMS by addressing analytes in a different mass range that overlaps with traditional MALDI. Experimental results from ME-SALDI IMS show substantially improved MS performance over conventional MALDI and SALDI
(C) 2009 Published by Elsevier Inc. on behalf of American Society for Mass Spectrometry. $1044-0305 / 09 / \$ 32.00$

doi:10.1016/j.jasms.2008.09.011
Published online September 20, 2008 Received June 24, 2008 Revised September 7, 2008 Accepted September 11, 2008 
methods in small-molecule detection, including reduced matrix interference and analyte fragmentation, expanded mass detection window to $10,000+$ amu, and much improved analyte ionization efficiency in smallfootprint, high-spatial resolution MS imaging.

\section{Experimental}

\section{Materials}

$\mathrm{N}$-type Sb-doped (100) single-crystalline silicon wafers at $0.005-0.02 \Omega / \mathrm{cm}$ were purchased from Silicon Sense, Inc. (Nashua, NH, USA) and stored under vacuum upon use. 1,2-Dimyristoyl-sn-glycero-3-phosphocholine (DMPC) was purchased from Avanti Polar Lipids, Inc. (Alabaster, AL, USA). L-Histidine, caffeine, 2,5dihydroxybenzoic acid (DHB), $\alpha$-cyano-4-hydroxycinnamic acid (CHCA), and 37\% formaldehyde solution were purchased from Sigma-Aldrich (St. Louis, MO, USA). Prednisone and methylene blue zinc chloride double salt were purchased from MP Biomedicals (Solon, $\mathrm{OH}$, USA). Hydrofluoric acid (HFA, 49\%), acetone, and $\mathrm{H}_{2} \mathrm{O}_{2}(30 \%)$ were purchased from Fisher Scientific (Pittsburgh, PA, USA). $\mathrm{CH}_{3} \mathrm{CH}_{2} \mathrm{OH}$ was purchased from Aaper Alcohol (Shelbyville, KY, USA). DI $\mathrm{H}_{2} \mathrm{O}$ of $18 \mathrm{M} \Omega$ (Millipore, Billerica, MA, USA) was used throughout the experiments. Insulin (bovine) and thioredoxin (Escherichia coli) were purchased from Applied Biosystems (Framingham, MA, USA). CD-1 female mouse heart tissues were fixed with $37 \%$ formaldehyde solution before the use. Mouse brain tissues were freshly frozen and stored at $-80{ }^{\circ} \mathrm{C}$ upon use.

\section{Porous Si Substrate Preparation}

Porous Si substrates were prepared as previously described [6, 21]. Briefly, 1- $\mathrm{cm}^{2}$ square-shaped Si wafer chips were dipped into a 5\% HFA solution in ethanol for $1 \mathrm{~min}$ to remove the oxidized layer first. These Si chips were then electrochemically etched in a $25 \%$ HFA/ethanol solution for $1 \mathrm{~min}$ at a current density of $5 \mathrm{~mA} / \mathrm{cm}^{2}$. White light from a 50-W tungsten lamp was used to irradiate silicon during etching. The produced porous $\mathrm{Si}$ substrates were then dipped into $15 \% \mathrm{H}_{2} \mathrm{O}_{2}$ for $1 \mathrm{~min}$, followed by a 1-min dip in 5\% HFA/ethanol solution to refresh the surface before analyte deposition.

\section{Sample Preparation for MS Measurements}

Analyte molecules were deposited on the solid surface either by the conventional drop-coating method or the spin-coating method. In the drop-coating method, a $0.5-\mu \mathrm{M}$ DMPC solution in acetone:ethanol (2:1), containing (or not) $10 \mathrm{mg} / \mathrm{mL}$ of CHCA, was drop-loaded onto porous silicon or a stainless steel MALDI target to compare the performance of MALDI, SALDI, and MESALDI. To demonstrate the extended mass range of ME-SALDI, a standard mix solution of $2.5 \mu \mathrm{M}$ insulin and $13.5 \mu \mathrm{M}$ thioredoxin in $10 \mathrm{mg} / \mathrm{mL}$ of CHCA was also loaded onto various substrates for MALDI, SALDI, and ME-SALDI comparison. In the spin-coating method, a 1-mM DMPC solution was prepared in acetone:ethanol (2:1) with or without $10 \mathrm{mg} / \mathrm{mL}$ of CHCA. Porous $\mathrm{Si}$ substrates (for ME-SALDI and SALDI) or flat Si substrates (for MALDI) were placed on a Laurell WS-400E6NPP-LITE spin coater (North Wales, PA, USA), followed by dropping $50 \mu \mathrm{L}$ of the DMPC solution with (for MALDI and ME-SALDI) or without (for SALDI) CHCA to the center of the substrates. The spin coater was then operated at $300 \mathrm{rpm}$ for $1 \mathrm{~min}$ that yielded a homogeneous DMPC/CHCA or a DMPC-only film, respectively. The average size of matrix crystals was $<5$ $\mu \mathrm{m}$ (Supplemental Material F1, which can be found in the electronic version of this article). Typically a lessthan-complete surface coverage was observed. In some cases, as specified in the text, only matrix molecules or matrix spiked with other internal standard molecules were deposited. Optical microscopic images were taken routinely to examine the quality of surface coatings with a DMRX light microscope (Leica Camera AG, Solms, Germany) equipped with a Donpisha XC-003P charge-coupled detector (CCD) camera (Leutron Vision AG, Glattbrugg, Switzerland).

\section{MS Instrument Parameters}

An Applied Biosystems Voyager DE-STR MALDI-timeof-flight (TOF) mass spectrometer was operated at an accelerating voltage of $20 \mathrm{kV}$. All experiments were operated in a linear mode except during brain MS imaging where the reflector mode was used. The laser intensity and delay time were varied to achieve optimal performance. An adjustable pinhole was placed close to the laser entrance window to adjust the diameter of the laser beam, although the laser size changed slightly as the laser input energy increased [22]. The largest measurable laser burn mark at each pinhole opening position was used to label the laser beam size (90 and 35 $\mu \mathrm{m})$ in the study. The laser radiation energy at various pinhole opening positions was measured outside of the sample chamber using a FieldMax-P laser energy meter (Coherent Inc., Santa Clara, CA, USA). Note the actual laser radiation energy on the substrate surface would be less; therefore the numbers sited in the following discussions serve only for relative comparison within this study. Twenty laser shots were fired to yield one MS spectrum and ten spectra were averaged to obtain one accumulated MS spectrum before further data analysis. At any given size of the laser beam, five MS replicates were collected at each laser irradiation energy level.

\section{Mass Spectrometry Imaging}

The mouse heart and brain tissue samples were mounted on a chunk with optical cutting temperature (OCT) compounds and ere sliced to 10 - to $40-\mu \mathrm{m}$-thick 
sections with a Cryo-cut microtome (American Optical Corp., Buffalo, NY, USA) at $-20^{\circ} \mathrm{C}$. The tissue sections were then transferred onto a porous silicon substrate. In the mouse heart experiment, a hydrophobic barrier surrounding the tissue section was drawn with an ImmEdge Pen (Vector Laboratories, Inc., Burlingame, CA, USA) to confine the solution during matrix deposition [23]. A mixture of small molecules, including L-histidine, phenylalanine, caffeine, and prednisone, were dissolved in a solution of $30 \mathrm{mg} / \mathrm{mL}$ of CHCA in ethanol:methanol:acetonitrile: $0.1 \%$ trifluoroacetic acid (water) $=2: 2: 1: 1$ as the internal standards. The matrix solution $(10 \mu \mathrm{L})$ was homogeneously spin-coated onto the heart tissue sample before MS measurements. In the mouse brain imaging experiment, matrix DHB was sublimated onto the $10-\mu \mathrm{m}$-thick tissue section at $110^{\circ} \mathrm{C}$ under vacuum for MS imaging [24]. Meanwhile, a similar section microtomed at the adjacent location was placed on a glass slide and stained with methylene blue. Limited by the view of the microscope, the optical images of stained brain sections were mosaics of several individual images in some cases. All tissue sections were imaged under a $35-\mu \mathrm{m}$ laser beam at $50-\mu \mathrm{m}$ steps. The MS instrument was controlled by the MALDI MS Imaging Tool software v2.2.0 (@ 2004 by Markus Stoeckli, Novartis Institutes for BioMedical Research, Basel, Switzerland). Twenty laser shots in mouse heart imaging and 50 shots in mouse brain imaging were averaged to yield one accumulated spectrum at each imaging pixel. The final ion maps, as well as the $y$-axis profiles, were reconstructed using BioMAP software (v3.7.5.4, Novartis).

\section{Results and Discussion}

Figure 1 illustrates the concept of hybrid ME-SALDI in which the analyte molecules were codeposited with matrix onto a porous silicon surface before MS measurements. DMPC was used as the model molecule in ME-SALDI, MALDI, and SALDI under individually optimized conditions, for its ease of ionization and fragmentation. The molecular ion peak $(\mathrm{m} / \mathrm{z}=678.5)$ and two major fragments $\left(\left[\mathrm{C}_{5} \mathrm{H}_{15} \mathrm{NPO}_{4}\right]^{+}, \mathrm{m} / z=184.1\right.$; $\left[\mathrm{C}_{5} \mathrm{H}_{12} \mathrm{~N}\right]^{+}, m / z=86.1$ ) of DMPC were clearly visible in ME-SALDI (Figure 1a). Only one matrix peak from $\mathrm{CHCA}-[\mathrm{CHCA}+\mathrm{Na}]^{+}$-was observed, yet with much lower ion intensity. In comparison, the low-mass region in MALDI was crowded with the matrix fragments and clusters (Figure 1b). Only the molecular ion peak of DMPC was unambiguously observed because it was located beyond the region of matrix background. SALDI detection showed similarly clean background in the low-mass region as in ME-SALDI, but the fragment ion peaks dominated and the molecular ion peak was indistinctive (Figure 1c), suggesting a harsher ionization condition.
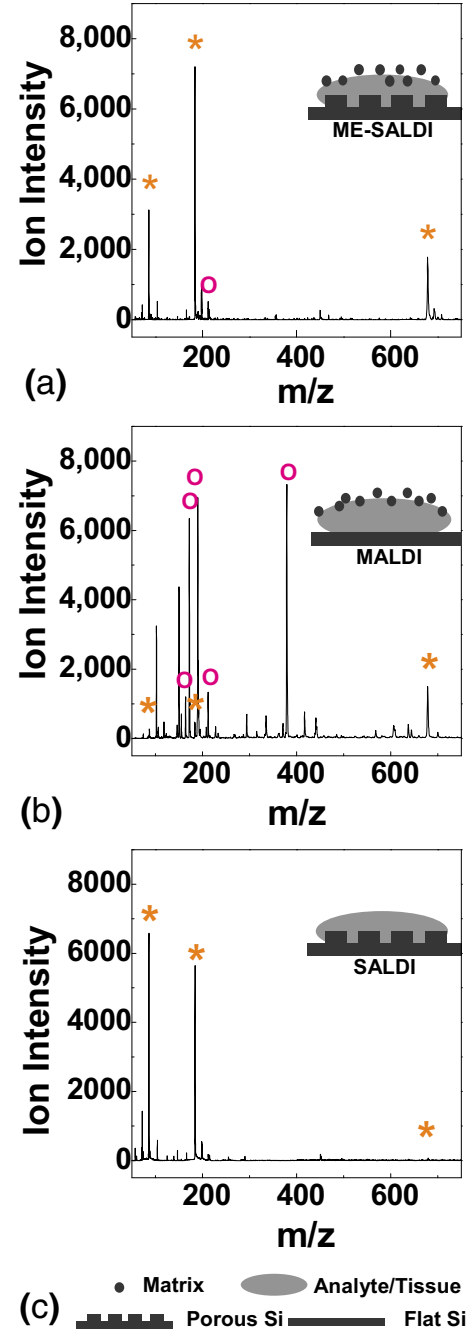

Figure 1. MS spectra of DMPC $(0.5 \mu \mathrm{M})$ using (a) ME-SALDI, (b) MALDI, and (c) SALDI. MS peaks associated with the analyte are labeled with asterisks $\left(^{*}\right)$ and the matrix peaks by circles $(\bigcirc)$. The insets were schematic drawings of each method. All data were collected under a $90-\mu \mathrm{m}$ laser beam.

\section{Reduced Matrix Interference and Analyte Fragmentation}

A clean and low-background MS spectrum in the lowmass region $(m / z<500)$ is essential in complex metabolite profiling. Matrix suppression effect scores (MSEs) were calculated using MSE $=\left\{\left(\sum I_{\text {[Analyte Ions }]}\right) /\right.$ $\left.\left(\sum I_{\text {[Analyte Ions] }}+\sum I_{\text {[Matrix Ions] }}\right)\right\}$ to quantify the influence of matrix interference [25]. As shown in Figure 2a, in MALDI the relative matrix signal intensities closely correlated to the laser energy input, varying from $<15 \%$ to a maximum of $75 \%$ when the laser energy was varied within the range of 2.4-3.9 $\mu \mathrm{J}$. In contrast, in ME-SALDI an average MSE score of $95 \pm 2 \%$ quantitatively confirmed the presence of cleaner mass spectra. The significantly improved MSE score in ME-SALDI is believed to be the result of selective enhancement of analyte ionization (Supplemental Material F2). It is also interesting to note that the MSE scores spanning across the broad 

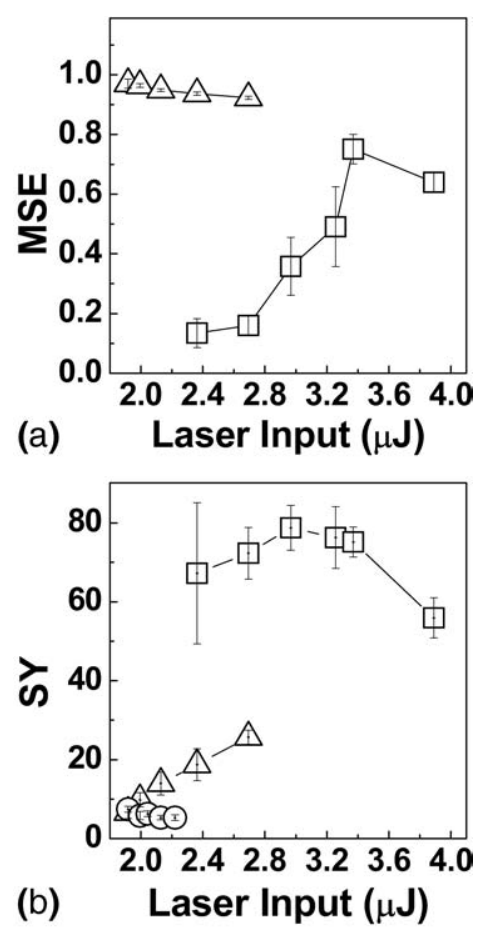

Figure 2. (a) MSE scores for DMPC detection using MALDI ( $\square$ ) and ME-SALDI $(\triangle)$ at different laser inputs. (b) The survival yields of DMPC molecular ion $(\mathrm{m} / \mathrm{z}=678.5)$ using MALDI $(\square)$, MESALDI $(\triangle)$, and SALDI $(\bigcirc)$ at different laser inputs. The error bars were calculated from five replicates.

range of laser irradiation energy studied $(1.8-2.6 \mu \mathrm{J})$ were relatively constant in ME-SALDI, a feature important in profiling unknown complex biological samples where little or no prior knowledge was available to allow optimization of laser fluence.

Although conventional SALDI also exhibits little background interference in the low-mass region, fragmentation often occurs as the result of destructive overheating during analyte desorption. The survival yields (SYs) of DMPC molecular ions in each ionization method were quantified as the relative intensity of the molecular ions: $S Y=\left\{\left(\sum I_{\text {[Molecular Ion] }]}\right) /\left(\sum I_{\text {[Molecular Ion] }}+\right.\right.$ $\left.\left.\sum I_{\text {[Fragment Ions] }}\right)\right\} \times 100 \%$. In SALDI, relatively low SYs were calculated because most DMPC molecules degraded and were detected in fragments (Figure 2b). A slight decrease was noticed with increasing laser inputs. In MALDI, on the other hand, analyte molecules were protected from direct photon excitation and destructive overheating. Subsequently, most molecules were detected intact and the average SY of the molecular ion was at $71 \pm 8 \%$, despite more intense laser irradiation was used. Note that the SY values calculated for MESALDI were at the same level as those in SALDI under low laser irradiation energy where MALDI matrix molecules were yet to be photoexcited. However, a steady increase in SY (i.e., less fragmentation) was observed under elevated laser energy in ME-SALDI, which was in drastic contrast to what was observed in conventional SALDI. This observation suggests the presence of a "softer" and a more "MALDI-like" mechanism in analyte ionization with increasing laser irradiation. Benefiting from this "softer" ionization process and improved ionization efficiency, the detectable mass range of ME-SALDI was easily extended to $>10,000 \mathrm{Da}$ compared with SALDI without compromising the qualities of MS signals, even when the irradiation laser fluence was kept below the matrix sublimation point (Supplemental Material).

Although the survival yield of DPMC in ME-SALDI was still lower than that in MALDI, the detection sensitivity of DMPC was nevertheless improved as the result of significantly enhanced overall ionization efficiency in ME-SALDI. The limits of detection (LODs) of DMPC molecular ions using three ionization sources under individually optimized experimental conditions were conservatively quantified as $0.01,0.05$, and $0.5 \mu \mathrm{M}$ (or 10, 50, and $500 \mathrm{fmol}$ ) for ME-SALDI, MALDI, and SALDI, respectively. A 5-fold improvement over MALDI and 50-fold improvement over SALDI were achieved with ease (Supplemental Material). Note that as a result of fragmentation of DMPC in SALDI and ME-SALDI, these LOD values calculated from DMPC molecular ion measurements were conservative estimates with the bias giving to the conventional MALDI technique. For example, at least one more order of magnitude improvement in LOD was achievable in ME-SALDI detection of DMPC if the major fragment ion $(m / z=184.1)$ was used in identification instead.

\section{Suggested ME-SALDI Mechanism}

Laser-induced analyte desorption and ionization constitute a complex process. Various theories have been presented to address the basic mechanisms underlying MALDI or SALDI [20, 26-29]. ME-SALDI, as a hybrid of MALDI and SALDI, is believed to share the energytransfer pathways of both. In particular, the laser threshold in ME-SALDI was found to be similar to that in SALDI but was significantly lower than that in MALDI (Figure 2b). This observation suggests the porous silicon surface-rather than the matrix moleculesbehaves as the energy-mediating center to effectively convert absorbed photons to local thermal energy and to facilitate desorption of analyte molecules; thus the coined name of ME-SALDI instead of surface-enhanced MALDI. During analyte desorption, the matrix molecules primarily serve as a carrying reagent that codesorb with analyte molecules through phase explosion, increasing the amount of analyte molecules in the gas phase and reducing the amount of energy directly transferred to the analyte molecules. Subsequently, more analyte ions (i.e., better sensitivity) and fewer fragments are detected. The control experiments where matrix molecules were deposited on a flat $\mathrm{Si}$ surface (as in conventional MALDI) did not show any appreciable signals of matrix or analyte molecules under the same low laser energy level, further confirming the critical 
role played by the porous structure of Si substrates in ME-SALDI desorption.

In addition to serving as the carrying agent, the matrix molecules play a pivotal role in analyte protonation. Three amino acids (valine, aspartic acid, and phenylalanine) of different gas-phase proton affinity (PA) were mixed with CHCA at the 1:1:1:3 $\mathrm{M}$ ratio for MALDI and ME-SALDI (Supplementary Material F3). In MALDI, consistent with their gas-phase PA, the ion signal intensities were similar for valine and aspartic acid, but both were weaker than that from phenylalanine. In ME-SALDI, an approximately 1.5-fold stronger ion signal was observed from valine, whereas the signal of aspartic acid reduced significantly to about $10 \%$ of the phenylalanine signal, consistent with the general trend of their $\mathrm{pI}$ values. These results suggest that unlike in MALDI where charge exchange occurs mostly in the gas phase, in ME-SALDI most analytes are probably ionized at the vacuum-substrate interface before and during desorption. Therefore, similar to SALDI, the condensed-phase acidity $\left(\mathrm{p} K_{a}\right)$ of an analyte seems to play a more important role, whereas gas-phase secondary ionization is limited because of the relatively low plume density $[19,20]$. The lack of proton donors that often leads to disappointing detection sensitivity in SALDI, however, is no longer an issue in ME-SALDI: acidic matrix molecules become the main source of protons in analyte ionization, as supported by the detection of significant enhanced matrix anions in the negative-mode detection (Supplemental Material F4). Previously observed reduction of matrix interference in ME-SALDI can also be rationalized as the result of a large portion of matrix ions were negatively charged species after donating the protons in ME-SALDI.

\section{Impact of Laser Beam Size}

A more pronounced improvement in detection sensitivity using ME-SALDI was realized during highresolution IMS where the use of a size-reduced laser beam was essential. The absolute ion currents from any laser-based MS ionization technique are expected to decrease when the laser beam size is reduced, primarily as a result of the loss of photon influx from beam blockage and fewer analyte molecules present in the field of analysis. An increase in the level of laser irradiation energy has been traditionally opted as the common remedy of the problem. However, in MALDI IMS, the increase of ion currents by increasing laser fluence slows down significantly as the laser beam size becomes smaller [27, 30]. When the laser beam was reduced to about $35 \mu \mathrm{m}$ using an iris, little improvement in the ion current was observed, even after the laser irradiation reached the upper limit of the instrument hardware output (Figure 3a). The laser size dependence of MALDI MS performance is suggested as the result of the changes in the projectile shape or the gas density within the desorption plume [26]. It is also likely related to faster dissipation of the absorbed
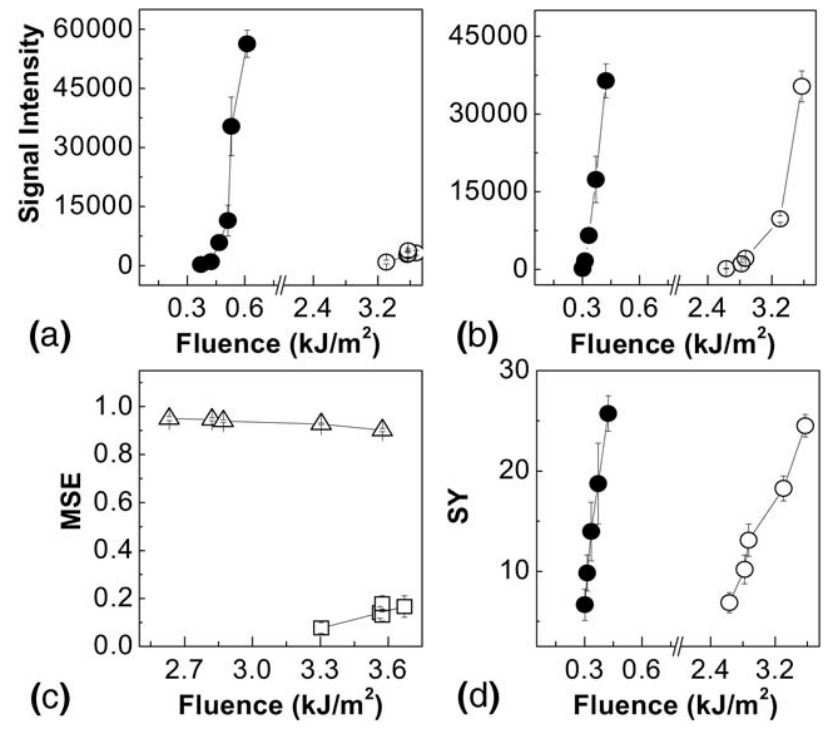

Figure 3. Ion intensities of the DMPC molecular ion in (a) MALDI and (b) ME-SALDI MS under the $90-\mu \mathrm{m}(\square)$ or 35- $\mu \mathrm{m}(\triangle)$ laser beam at different laser fluences. (c) The MSE scores of DMPC detection using MALDI $(\square)$ and ME-SALDI $(\triangle)$ under the 35- $\mu \mathrm{m}$ size laser beam. (d) The survival yields of the DMPC molecular ion using ME-SALDI under the 90- $\mu \mathrm{m}(\square)$ and 35- $\mu \mathrm{m}(\triangle)$ size laser beam. The error bars were calculated from five replicates.

energy to the surrounding environment under the smaller laser beam. In SALDI, however, ionization mainly occurs on the porous silicon surface, which is less dependent on the plume geometry and density. Furthermore, the nanoporous structure of the SALDI substrate $(\sim 50 \mathrm{~nm} \ll 35 \mu \mathrm{m}$ laser beam) effectively "confines" the absorbed energy locally, independent of the size of the laser beam used [31, 32]. Therefore, comparable MS performance was achievable in SALDI even when the laser beam was reduced, albeit highenergy input is needed to make up the overall flux (data not shown). Given that ME-SALDI shares the same condensed-phase ionization pathway as that of SALDI, the MS response is expected and was indeed found to be less dependent on the beam size as well (Figure 3b).

The previously described benefits of ME-SALDI over MALDI and SALDI were also exemplified at the reduced laser size: a relatively unchanged MSE score was calculated in ME-SALDI at $93 \pm 2 \%$, 7-fold higher than that of MALDI $(14 \pm 4 \%)$, under the $35-\mu \mathrm{m}$ laser beam (Figure 3c). Unlike that under the 90- $\mu \mathrm{m}$ laser beam, the background interference observed in MALDI can hardly be improved over the range of laser flux. On the other hand, the increased laser energy input at the reduced laser size did not necessarily result in a decreased SY of molecular ion in ME-SALDI. As shown in Figure 3d, similar SYs were calculated, which demonstrated relatively independent analyte fragmentation to the laser beam size in ME-SALDI. Together, compared with conventional MALDI and SALDI, ME-SALDI exhibits reduced dependence on laser beam sizes. Along with its reduced matrix interference, reduced fragmen- 

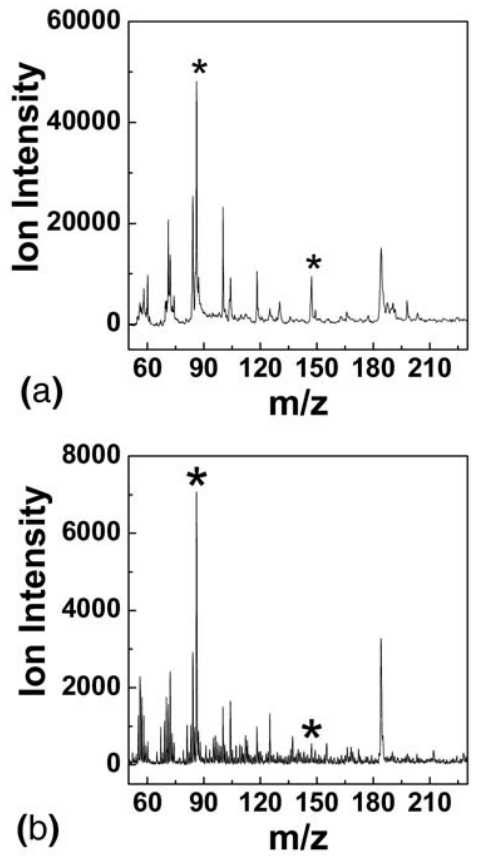

Figure 4. Mouse heart tissue sections of $40-\mu \mathrm{m}$ thickness were placed onto porous/flat silicon surface and immersed into a $1 \mathrm{mM}$ L-lysine solution in $2 \mathrm{mg} / \mathrm{mL}$ CHCA. The signal intensity of L-lysine detected from the tissue atop of (a) a porous silicon substrate in ME-SALDI was about 20-fold stronger than that from (b) the flat silicon in MALDI. The ion intensity of the $\mathrm{m} / \mathrm{z}=86.1$ peak was about 7-fold stronger.

tation, and improved detection sensitivity, ME-SALDI is shown to potentially provide the best overall MS performance for high-resolution 2-D IMS.

\section{MS Imaging of Mouse Tissue Sections}

Although there was no physical contact between analytes atop of the tissue section and the porous silicon underneath the tissue, direct imaging of small biomolecules with desorption ionization on silicon (DIOS)-MS has been reported [6]. The photon energy absorbed by porous silicon was likely converted to thermal energy and then transferred to the top layer of the tissue surface in the desorption/ionization process. To confirm the feasibility of using ME-SALDI in 2-D imaging, tissue sections of 10, 20,30, and $40 \mu \mathrm{m}$ in thickness were placed on a porous or a flat silicon surface side by side, followed by coating a standard molecule-lysine-atop to ensure the same amount of analyte molecules was examined. Compared with those from a flat surface in the conventional MALDI mode, it is astonishing to see that much stronger MS signals from the tissue placed atop the porous surface (i.e., in the ME-SALDI mode) were observed, in spite of the $40-\mu \mathrm{m}$-thick tissue section between the surfaces (Figure 4). The exact cause behind this enhancement in the presence of a thick insulating layer is not understood at the moment, although the benefit of using ME-SALDI in tissue imaging is apparent.
The use of ME-SALDI in MS imaging under a reduced laser beam was examined using mouse brain and heart tissues. Figure $5 \mathrm{~b}$ shows an optical image of the mouse heart tissue collected before deposition of a thin layer of CHCA matrix atop. The tissue was purposely placed on the border of a porous silicon substrate such that half of the tissue rested on the porous substrate to be imaged in the ME-SALDI mode, whereas the other half was on the flat Si surface to be imaged in the conventional MALDI mode. The blue dotted line outlined the boundary of the tissue sample and the virtual boundary of the porous surface where the tissue atop the porous Si portion showed a darker shade in the picture. This experimental setup allowed direct side-by-side performance comparison of two MS ionization methods and eliminated any ambiguity introduced by using different tissue samples. MS spectra were collected with a $35-\mu \mathrm{m}$ laser beam at $50-\mu \mathrm{m}$ scanning steps. Although the experimental conditions were optimized for MALDI IMS whose performance was more sensitive to instrumental parameters and required higher laser irradiation energy, stronger overall signal intensities were observed in the representative spectrum of ME-SALDI (Figure $5 c$ versus a). Figure $5 \mathrm{~d}-\mathrm{f}$ show the reconstructed ion maps of total ions or selective species. The species at $\mathrm{m} / \mathrm{z} 86.1$ was likely from the headgroup of phosphatidylcholine and that at $\mathrm{m} / \mathrm{z}$ 359.2 was an internal standard prednisone spiked into the matrix solution before matrix deposition. Stronger ion intensities were universally observed from the left bottom section of the tissue atop the porous silicon than those from the right top half of the tissue on the flat surface. The exception is for matrix ions where much smaller differences were observed between MALDI and ME-SALDI for ions at $m / z 190.1$, i.e., [CHCA H]. A linear-scanning profile for each ion map was carried out along the red dotted line with a yellow star indicating the border of two surfaces (the right side of the yellow star corresponds to the flat portion). A clear difference in average ion intensity was observed between MESALDI and MALDI for Figure $5 \mathrm{~h}-\mathrm{j}$, but Figure 5k of the matrix ion showed little difference.

To quantify the degree of improved MS imaging performance, roughly 1800 spectra were averaged for both MS ionization methods. Statistic calculation shows that an average of a nearly 20 -fold improvement for detection of seven representative analytes was achieved in ME-SALDI imaging to MALDI. Meanwhile, the matrix background exhibited an average of only 1.5 -fold enhancement (for three matrix-related ion peaks) to that of MALDI imaging, confirming again the reduced matrix interference. A higher degree of improvement was obtained when 2-D IMS was conducted under the conditions more suitable for ME-SALDI (Supplementary Material F5).

The capability of ME-SALDI IMS in visualizing distribution of natural biomolecules was further demonstrated in the examination of mouse brain tissues. Figure $6 \mathrm{a}$ and $\mathrm{g}$ show the optical images of the coronal 


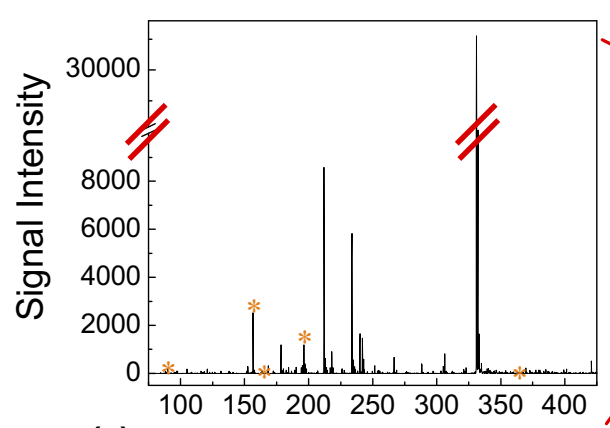

(a)

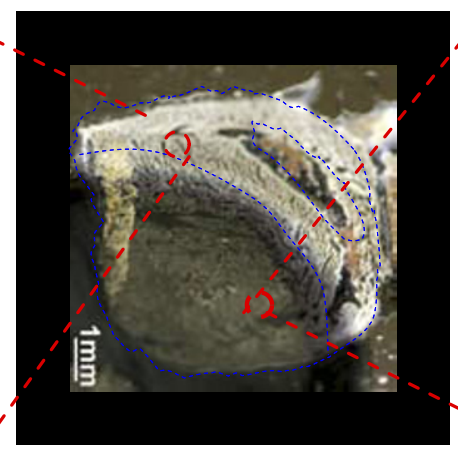

(b)

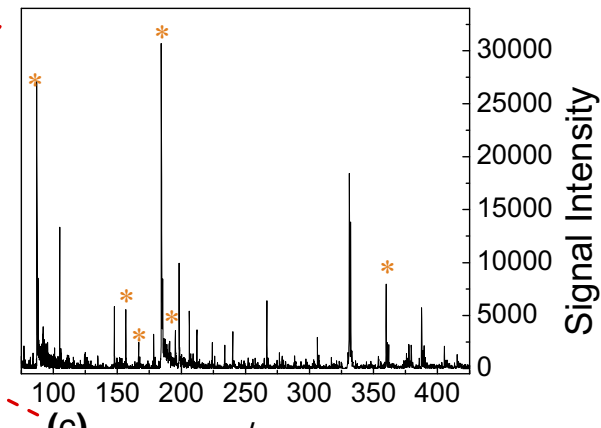

(c) $\mathrm{m} / \mathrm{z}$

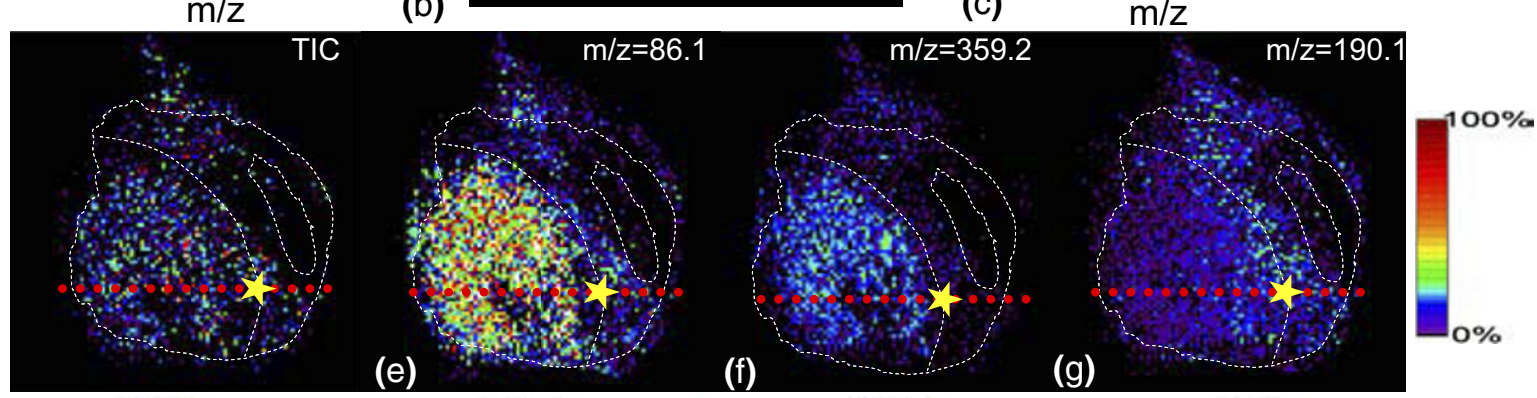

(d)

(t)

(g)

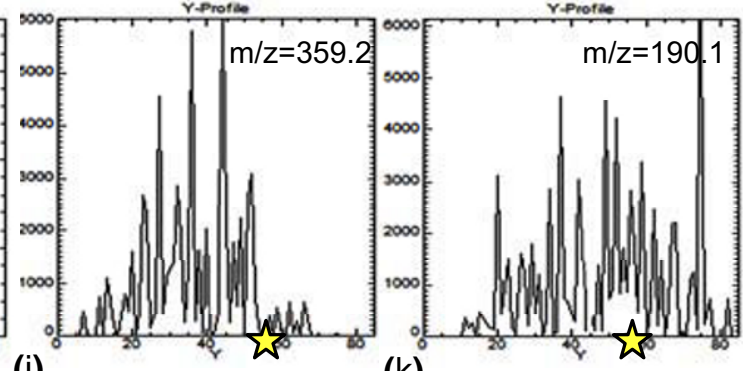

(h)

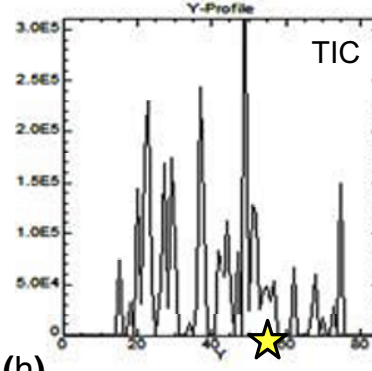

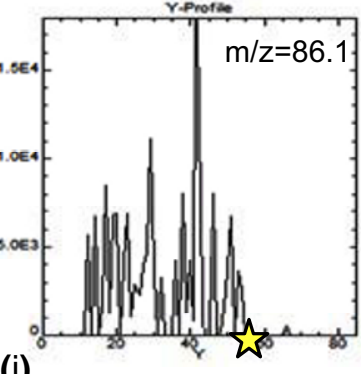

(i) (j) (k)

Figure 5. Representative MS spectra collected from the tissue atop of (a) the flat silicon in MALDI and (c) the porous silicon in ME-SALDI. MS peaks associated with the phosphocholine headgroups and the internal standards are labeled with asterisks $\left(^{*}\right)$. (b) An optical image of a CB1 mouse heart tissue section placed half inside/half outside of a porous silicon substrate. The blue-dotted line shows the virtual boundary of two surfaces and the outline of the tissue. Small tissue damage in the right middle portion was blocked by a hydrophobic barrier. The reconstructed 2-D images for (d) total ion chromatogram (TIC) and the ions at (e) $m / z=86.1$, (f) $\mathrm{m} / \mathrm{z}=359.2$, and (g) $\mathrm{m} / \mathrm{z}=190.1$ are representative ion maps. Panels $\mathbf{h}, \mathbf{i}, \mathbf{g}$, and $\mathbf{k}$ are the scanning profiles in the corresponding MS images at the location indicated by the red dotted lines. The yellow star indicates the border where the porous and the flat surface underneath the tissue met along the red line.

sections of mouse cerebrum and mouse cerebellum, respectively. Clear detection of cholesterol and phospholipids was evident in the MS spectra (Figure $6 \mathrm{~b}$ and h). The 2-D reconstructed ion maps show that cholesterol and certain phospholipids were differentially expressed at specific regions of mouse brain. As shown in Figure $6 \mathrm{c}$ and i, the cholesterol ions $\left[\mathrm{M}-\mathrm{H}_{2} \mathrm{O}+\mathrm{H}\right]^{+}$ $(\mathrm{m} / \mathrm{z}=369.4)$ were found highly concentrated in the white matter at corpus callosum and cerebellum. In contrast, the adducts of phosphocholine (PC) 32:0 ( $\mathrm{m} / \mathrm{z}=$ 772.6) and PC 38:6 $(\mathrm{m} / \mathrm{z}=844.5)$ were complementarily distributed in cerebral cortex and striatum, but almost absent in corpus callosum (Figure 6d and f). The PC 40:4 molecular ions $[\mathrm{M}+\mathrm{H}]^{+}(\mathrm{m} / \mathrm{z}=838.6)$ were concentrated in corpus callosum, brain stem, and the cerebellar nuclei, but absent in the molecular layer of cerebellum (Figure 6e and 1). As shown in Figure 6j, the potassium adducts of sphingomyelin (SM) 18:0 $(\mathrm{m} / \mathrm{z}=769.6)$ were highly expressed in the molecular layer of cerebellum but barely in brain stem, complementarily to the spatial distribution of PC 36:1 and PC 40:4 in the gray matter. These findings were consistent with the reported histological and traditional MS studies [33, 34], and it is the first time that the complementary spatial distribution of cholesterol and whole lipids was visualized simultaneously using a laser-based MS imaging method.

\section{Conclusions}

In summary, we have demonstrated that by synergistically combining the traditional MALDI and SALDI methods, the hybrid ME-SALDI ionization method provides an attractive approach to imaging small molecules under a reduced laser beam. By separating the 
(a)

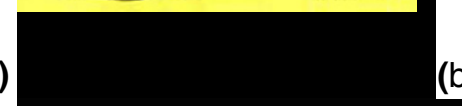

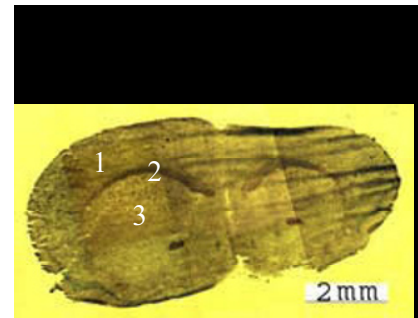

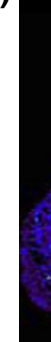

(b)

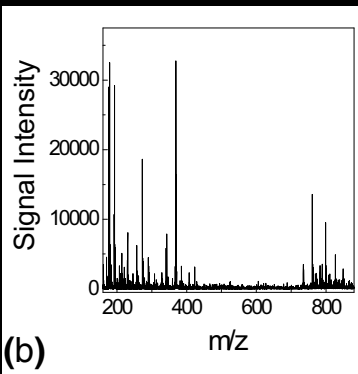

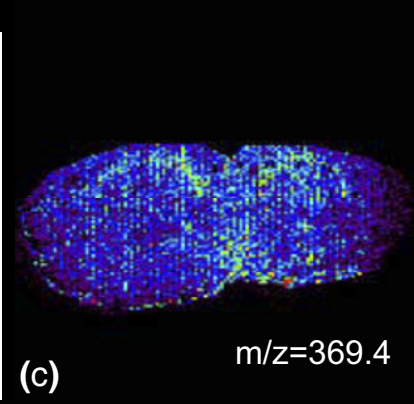

(d)

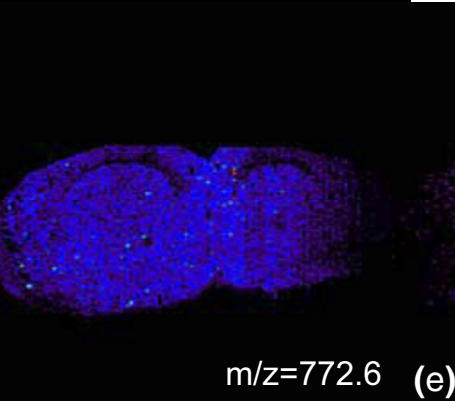

(c) $\mathrm{m} / \mathrm{z}=369.4$ (g)

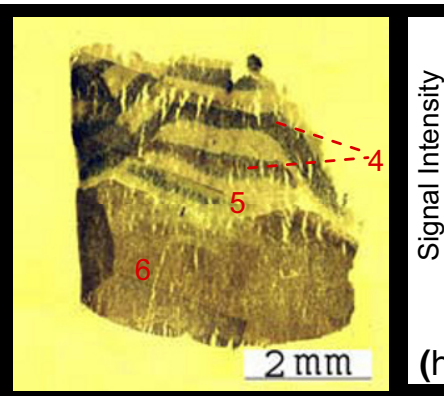

(h)

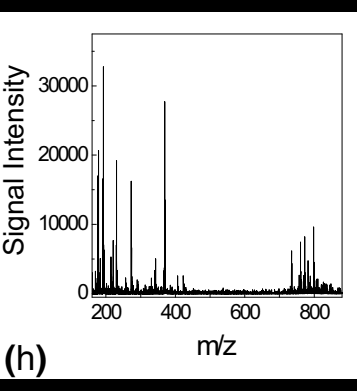

(i)

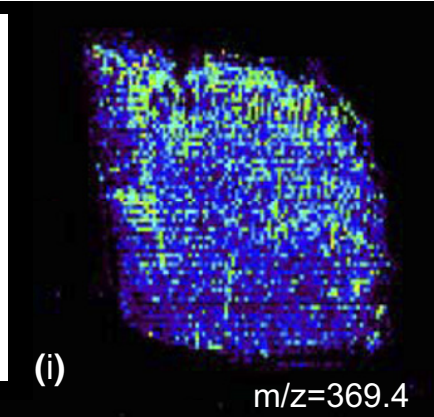

(j)
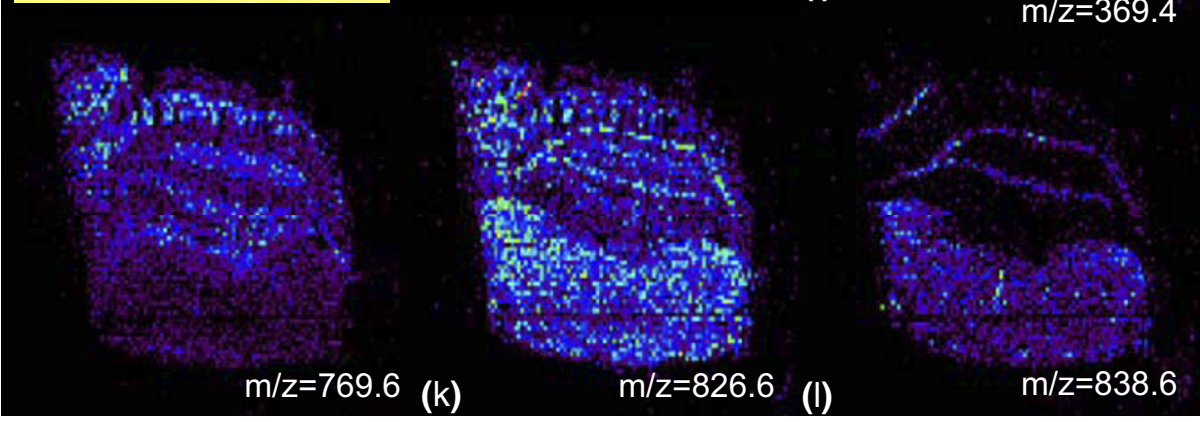

Figure 6. Optical images of the coronal sections of (a) mouse cerebrum and (g) mouse cerebellum. Representative MS spectra collected from the adjacent coronal sectioning of (b) mouse cerebrum and (h) mouse cerebellum in Me-SALDI, respectively. Also shown are reconstructed 2-D images for ions at (c) $m / z=369.4$, (d) $m / z=772.6$, (e) $m / z=838.6$, and (f) $m / z=844.5$ from the mouse cerebrum coronal section, and for ions at (i) $m / z=369.4,(\mathbf{j}) \mathrm{m} / z=769.6,(\mathbf{k}) \mathrm{m} / z=826.6$, and $(\mathbf{l}) \mathrm{m} / \mathrm{z}=838.6 \mathrm{from}$ mouse cerebellum coronal section. For molecular identification see the text. Different areas in the brain tissue are labeled numerically: 1, cerebral cortex; 2, corpus callosum; 3, striatum; 4, cerebellar nuclei; 5 , molecular layer in cerebellum; 6 , brain stem.

primary photon-absorption medium (i.e., porous surfacefacilitated desorption) from the primary protonation step (i.e., matrix-assisted proton transferring), MESALDI enables independent optimization of desorption and ionization processes to achieve the best imaging outcome. The demonstrated simultaneous imaging of cholesterol and lipids in mouse brain tissue opens opportunities in further investigation of their roles in brain development and damage repairs.

\section{Acknowledgments}

We thank Dr. Dykstra at the Laboratory for Advanced Electron and Light Optical Methods at College of Veterinary Medicine, North Carolina State University (NCSU) for tissue preparation. We appreciate Drs. G. M. Pollack, J. Padowski and W. Yue at School of Pharmacy, University of North Carolina at Chapel Hill, and Mrs. Welker at College of Agriculture and Life Sciences at NCSU for providing mice tissue samples. We are also thankful to Dr. M. Stoeckli at Novartis Institutes for BioMedical Research, 
Basel, Switzerland for the help with MS imaging software. This work was partially supported by Canon, Inc. and an NCSU FPRD award.

\section{References}

1. Caprioli, R. M.; Farmer, T. B.; Gile, J. Molecular Imaging of Biological Samples: Localization of Peptides and Proteins Using MALDI-TOF MS. Anal. Chem. 1997, 69, 4751-4760.

2. Ostrowski, S. G.; Van Bell, C. T.; Winograd, N.; Ewing, A. G. Mass Spectrometric Imaging of Highly Curved Membranes During Tetrahymena Mating. Science 2004, 305, 71-73.

3. Altelaar, A. F. M.; Van Minnen, J.; Jimenez, C. R.; Heeren, R. M. A.; Piersma, S. R. Direct Molecular Imaging of Lymnaea stagnalis Nervous Tissue at Subcellular Spatial Resolution by Mass Spectrometry. Anal. Chem. 2005, 77, 735-741.

4. Drexler, D. M.; Garrett, T. J. Cantone, J. L · Diters, R. W · Mitroka, J. G. Prieto Conaway, M. C.; Adams, S. P.; Yost, R. A.; Sanders, M. Utility of Imaging Mass Spectrometry (IMS) by Matrix-Assisted Laser Desorption Ionization (MALDI) on an Ion Trap Mass Spectrometer in the Analysis of Drugs and Metabolites in Biological Tissues. J. Pharmacol. Toxicol. Methods 2007, 55, 279-288.

5. Nemes, P.; Vertes, A. Laser Ablation Electrospray Ionization for Atmospheric Pressure, in Vivo, and Imaging Mass Spectrometry. Anal. Chem. 2007, 79, 8098-8106.

6. Liu, Q.; Guo, Z.; He, L. Mass Spectrometry Imaging of Small Molecules Using Desorption/Ionization on Silicon. Anal. Chem. 2007, 79, 35353541.

7. Cha, S.; Yeung, E. S. Colloidal Graphite-Assisted Laser Desorption/ Ionization Mass Spectrometry and $\mathrm{MS}^{n}$ of Small Molecules. 1. Imaging of Cerebrosides Directly from Rat Brain Tissue. Anal. Chem. 2007, 79, 2373-2385.

8. Cornett, D. S.; Reyzer, M. L.; Chaurand, P.; Caprioli, R. M. MALDI Imaging Mass Spectrometry: Molecular Snapshots of Biochemical Systems. Nat. Methods 2007, 4, 828-833.

9. $\mathrm{Wu}, \mathrm{K}$. J.; Odom, R. W. Matrix-Enhanced Secondary Ion Mass Spectrometry: A Method for Molecular Analysis of Solid Surfaces. Anal. Chem. 1996, 68, 873-882

10. Cooks, R. G.; Ouyang, Z.; Takats, Z.; Wiseman, J. M. Ambient Mass Spectrometry. Science 2006, 311, 1566-1570

11. Shiea, J.; Huang, M.-Z.; Hsu, H.-J.; Lee, C.-Y.; Yuan, C.-H.; Beech, I.; Sunner, J. Electrospray-Assisted Laser Desorption/Ionization Mass Spectrometry for Direct Ambient Analysis of Solids. Rapid Commun. Mass Spectrom. 2005, 19, 3701-3704.

12. Coon, J. J.; Steele, H. A.; Laipis, P. J.; Harrison, W. W. Laser DesorptionAtmospheric Pressure Chemical Ionization: A Novel Ion Source for the Direct Coupling of Polyacrylamide Gel Electrophoresis to Mass Spectrometry. J. Mass. Spectrom. 2002, 37, 1163-1167.

13. Sampson, J. S.; Hawkridge, A. M.; Muddiman, D. C. Generation and Detection of Multiply-Charged Peptides and Proteins by MatrixAssisted Laser Desorption Electrospray Ionization (MALDESI) Fourier Transform Ion Cyclotron Resonance Mass Spectrometry. I. Am. Soc. Mass Spectrom. 2006, 17, 1712-1716.

14. Tanaka, K.; Waki, H.; Ido, Y.; Akita, S.; Yoshida, Y.; Yohida, T. Protein and Polymer Analyses Up to $m / z 100,000$ by Laser Ionization Time-ofFlight Mass Spectrometry. Rapid Commun. Mass Spectrom. 1988, 2, 151-153.

15. Sunner, J.; Dratz, E.; Chen, Y.-C. Graphite Surface-Assisted Laser Desorption/Ionization Time-of-Flight Mass Spectrometry of Peptides and Proteins from Liquid Solutions. Anal. Chem. 1995, 67, 4335-4342.
16. Dale, M. J.; Knochenmuss, R.; Zenobi, R. Graphite/Liquid Mixed Matrixes for Laser Desorption/Ionization Mass Spectrometry. Anal. Chem. 1996, 68, 3321-3329.

17. Dale, M.; Knochenmuss, R.; Zenobi, R. Two-Phase Matrix-Assisted Laser Desorption/Ionization: Matrix Selection and Sample Pretreatment for Complex Anionic Analytes. Rapid Commun. Mass Spectrom. 1997, 11, 136-142.

18. Zhou, H.; Xu, S.; Ye, M.; Feng, S.; Pan, C.; Jiang, X.; Li, X.; Han, G.; Fu, Y.; Zou, H. Zirconium Phosphonate-Modified Porous Silicon for Highly Specific Capture of Phosphopeptides and MALDI-TOF MS Analysis. J. Proteome Res. 2006, 5, 2431-2437.

19. Liu, Q.; He, L. Quantitative Study of Solvent and Surface Effects on Analyte Ionization in Desorption Ionization on Silicon (DIOS) Mass Spectrometry. J. Am. Soc. Mass Spectrom. 2008, 19, 8-13.

20. Chen, C.; Chen, H.; Aleksandrov, A.; Orlando, T. M. Roles of Water Acidity, and Surface Morphology in Surface-Assisted Laser Desorption/Ionization of Amino Acids. J. Phys. Chem. C 2008, 112, 6953-6960.

21. Finkel, N. H.; Prevo, B. G.; Velev, O. D.; He, L. Ordered Silicon Nanocavity Arrays in Surface-Assisted Desorption/Ionization Mass Spectrometry. Anal. Chem. 2005, 77, 1088-1095.

22. Chaurand, P.; Schriver, K. E.; Caprioli, R. M. Instrument Design and Characterization for High Resolution MALDI-MS Imaging of Tissue Sections. J. Mass Spectrom. 2007, 42, 476-489.

23. Agar, N. Y. R.; Yang, H. W.; Carroll, R. S.; Black, P. M.; Agar, J. N. Matrix Solution Fixation: Histology-Compatible Tissue Preparation for MALDI Mass Spectrometry Imaging. Anal. Chem. 2007, 79, 7416-7423.

24. Hankin, J.A.; Barkley, R.M.; Murphy, R. C. Sublimation as a Method of Matrix Application for Mass Spectrometric Imaging. J. Am. Soc. Mass Spectrom. 2007, 18, 1646-1652.

25. McCombie, G.; Knochenmuss, R. Small-Molecule MALDI Using the Matrix Suppression Effect to Reduce or Eliminate Matrix Background Interferences. Anal. Chem. 2004, 76, 4990-4997.

26. Dreisewerd, K. The Desorption Process in MALDI. Chem. Rev. 2003, 103 , 395-426.

27. Knochenmuss, R. Ion Formation Mechanisms in UV-MALDI. Analys 2006, 131, 966-986.

28. Alimpiev, S.; Grechnikov, A.; Sunner, J.; Karavanskii, V.; Simanovsky, Y.; Zhabin, S.; Nikiforov, S. On the Role of Defects and Surface Chemistry for Surface-Assisted Laser Desorption Ionization from Silicon. J. Chem. Phys. 2008, 128, 014711.

29. Luo, G.; Chen, Y.; Daniels, H.; Dubrow, R.; Vertes, A. Internal Energy Transfer in Laser Desorption/Ionization from Silicon Nanowires. J. Phys. Chem. B 2006, 110, 13381-13386.

30. Dreisewerd, K.; Schurenberg, M.; Karas, M.; Hillenkamp, F. Influence of the Laser Intensity and Spot Size on the Desorption of Molecules and Ions in Matrix-Assisted Laser Desorption/Ionization with a Uniform Beam Profile. Int. J. Mass Spectrom. Ion Processes 1995, 141, 127-148.

31. Schuerenberg, M.; Dreisewerd, K.; Hillenkamp, F. Laser Desorption/ Ionization Mass Spectrometry of Peptides and Proteins with Particle Suspension Matrixes. Anal. Chem. 1999, 71, 221-229.

32. Alimpiev, S.; Nikiforov, S.; Karavanskii, V.; Minton, T.; Sunner, J. On the Mechanism of Laser-Induced Desorption-Ionization of Organic Compounds from Etched Silicon and Carbon Surfaces. J. Chem. Phys. 2001, 115, 1891-1901.

33. Puolitaival, S. M.; Burnum, K. E.; Cornett, D. S.; Caprioli, R. M Solvent-Free Matrix Dry-Coating for MALDI Imaging of Phospholipids. J. Am. Soc. Mass Spectrom. 2008, 19, 882-886.

34. Sjovall, P; Johansson, B.; Lausmaa, J. Localization of Lipids in FreezeDried Mouse Brain Sections by Imaging TOF-SIMS. Appl. Surf. Sci. 2006, 252, 6966-6974. 